\title{
A Preliminary Study on the identification, Evolution and Protection of Historical Geographic Names in China
}

\author{
Ye Rong ${ }^{\mathrm{a}}$, Fan Jinzhao ${ }^{\mathrm{b},}$ * \\ Department of Geography, School of Earth Sciences, Zhejiang University, Hangzhou, China \\ a11638001@zju.edu.cn, bzfan@zju.edu.cn.
}

Keywords: historical geographic names; word; feature; evolution; protection

Abstract: Historical geographic names belong to geographic names, which divide into the names that are still in use and those disappeared. Due to natural elements or human elements, historical geographic names continuously evolved. So far, there are six different types. In consideration of their significances, it is high time to protect historical geographic names. As for the protections, it should be based on the types of the evolution of the historical geographic names.

\section{Introduction}

Historical geographic names are the products of history, some are still in use today, their meaning are not much changed still maintaining vitality; Some have changed their contents, others have faded into the past completely abandoned and no longer used. The ancient geographic names record the rise and fall of the country, the past of the nation, the developments of the society and even the changes of the natural environment. They witness the history. Historical geographic names are the most direct memories of the hometown for the old people. If they do not have a full understanding of these old places, once they are renamed they will lose their hometown. Thus, studying historical geographic names can not only understand the historical evolution and the cultural connotation, but also clearly acknowledge the political, social, natural and other development conditions of a certain historical period, providing reference for today's developments. More importantly, historical geographic names are also solid weapons for defending the territory and the old names on record are the most direct proofs of territorial sovereignty today. Therefore, the protection of historical geographic names is urgent.

The study of historical geographic names has been discussed by scholars in China since the 1980s. In 1983, Professor Chen Qiao-yi pointed out that the historical geographic names in Zhejiang Province were originated from the dialect of ancient Wuyue language, which could not be interpreted in a far-fetched way in Mandarin. [1] In 1989, Mr. Yin Jun-ke firstly put forward the concept of "historical geographic names" and its importance in the study of toponymy, he stated: "History place names are names that existed in old times and disappeared in today ".He also took Beijing in different historical geographical entities, such as Ji County, Yongding River, Huo County as examples. This paper discussed the evolution of these geographic names, pointed out that history geographic names are one of the main researches of Toponymy. [2] In 1998, Mr. Yan Zhi-jie proposed that "historical geographic names are of great significances for people's daily 
communication and social developments and they are witnesses of times changes and have practical and strategic importance for social developments" but he did not clearly explain what historical geographic names are. [3] In 2000, Mr. Chen Qiao-yi came up with three general aspects of toponym research since the Pre-Qin Dynasty including geographical location researches, evolution change researches and origin history researches. He called on contemporary toponym workers to sort out and develop on the basis of previous achievements. [4][5] In 2005, Mr. He Ling analyzed the status of historical toponym in western regions, its profound history and the importance of protecting historical toponym in western regions. [6] In 2009, Mr. Yue Sheng-yang and Mr. Yu Chang-hong clearly proposed the definitions of "historical geographic names", differing from Mr. Yin Jun-ke, he believed that: "historical geographic names are places formed before a certain years, which in mainland China mainly refer to places formed before 1949. Historical geographic names include those that are in use and those that are not." It is pointed out that historical geographic names are an important part of Beijing's historical and cultural city heritage. It is a must to establish the protection principle and plan for historical geographic names. [7] In 2011, Mr. Wu Peng-fei took Kaifeng City as an example and raised several countermeasures for the protection of historical geographic names in the process of urban modernization. [8] In 2013, Mr. Lin Yu-jun and Mr. Zhang Qing-hua presented several major reasons for the constant disappearance of historical geographic names and several measures for the utilization and protection of historical geographic names. [9]

Summarized from the study of historical geographic names, there are still some discussions, mainly: (1) There are different views of the definition of historical geographic names. Generally speaking, which is the correct time for the historical geographic name. Also, it is controversial whether the geographic names that are still in use from historical period are the historical geographic names or not; (2) There is no systematic review or summary of the evolution mode of historical geographic names; (3) Due to the lack of preparation of these theories, policies on the protection and management of historical geographic names are also lack of pertinence and effectiveness. Therefore, this study mainly aims at these three aspects and tries to make a preliminary discussion on the definition, evolution and protection of historical geographic names in China.

\section{The Identification Of Historical geographic Names In China}

Geographic name, in short, is "place" (that is, all kinds of "area") or the name of the "location" (that is, all kinds of natural, human geographic "entity") or the name of the meaning" geographic name is the distinguished name given by the geographic area through specific location, regional range of geographic entity, which has propriety and sociality" [10]. The origin of geographic names is from people's understanding of natural environment and human social activities. Geographic names are a linguistic phenomenon. Given the long history of mankind, geographic names can change several times over time with the evolution of language, the migration of inhabitants, the change of dynasties and the revolution of politics. The constant changes of geographic names will inevitably result in the division between the current geographic names and the past ones and between the old and the new ones [3].

At present, there are mainly two opinions on the definition of historical geographic names as mentioned above. One is Mr. Yin Jun-ke's view that historical geographic names are "places that existed in the past but have disappeared and not used in nowadays" [2]. Another is Mr. Yue Sheng-yang and some other scholars' suggestion "historical geographic names refer to places formed before a certain number of years, in the Chinese mainland mainly refer to places formed before 1949. Historical geographic names include those that are in use and those that are not"[7]. 
This study agrees with the latter. There are two main reasons for this:

Firstly, Mr. Yin Jun-ke posed that "names existed in the past but have disappeared now" belong to historical names which is too narrow and harsh. If so, historical geographic names are very limited in number; at the same time, a large number of geographic names appeared in historical periods are still in use. However, there is no essential difference in the rules of their generation and development between geographic names which are no longer used or still used and their protection. To some extent, the geographic names raised in historical periods that are still in use may be of higher value.

Secondly, it is difficult to define a geographic name as "not used". Due to the changes of various factors, the usage of geographic names is very complex. The word of geographic name might be used in different places. That is, in the beginning it is a political geographic name. After removing, renaming or any other changes, the old names are no longer used in the same level of administrative zones, but they might become the name of low level administrative zones, community, village, or even road; if this situation is considered to be out of use, it is difficult to define historical geographic names.

Therefore, this study agrees with the views of Mr. Yue Sheng-yang, that is, the so-called "historical geographic names" refers to all kinds of geographic names that appeared in historical periods, especially the places such as political districts, settlement geographic names, natural landscape geographic names, and urban street names. In terms of the time limit, it can be roughly defined as 1949. The names appeared or used before 1949 belong to historical geographic names, regardless of whether they are currently used or not. After 1949, the emergence of geographic names can be considered as modern, contemporary geographic names. Of course, the lower limit of historical geographic names will change over time. For example, the geographic names before 1979 have some historical significance; therefore, some can or will be included in the historical geographic names.

As the product of history, historical geographic names have been inherited from generation to generation and have their own cultural identity and continuity. In particular, the geographic names that continue existing with a long history, with profound historical culture, unique geographical culture and plain local cultural connotation are worth cherishing. The most valuable geographic names in history are likely to be listed in a certain level of protection or national legal types of geographic names heritage and to become local, national, and even world's common wealth.

\section{Evolution of Historical Geographic Names in China}

There are few systematic reviews on the evolution of historical geographic names in existing literatures, and the most relevant research focus on the status, function and protection measures of historical geographic names. This study believes that in order to analyze the evolution of historical geographic names, we should start from the change of the relationship between "Name" and "Entity" of historical geographic names. "Name" itself is the core of the geographic name and the carrier of its implication; In addition to the literal meaning, the word actually has the meaning of a specific referential place, that is, it has become a whole (" proper noun ") and a conceptual symbol of a place, which is the more important meaning of the word. "Entity" refers to the objective geographic entity or geographic region, and all kinds of natural, historical, social and cultural information contained within it. The evolution of historical geographic names is closely related to the changes of the relationships between them. From the differences between the changes of these two relations, the evolution of historical geographic names can be divided into six different situations (as shown in FIG. 1, circle refers to solid space, square refers to geographic names, solid line refers to no change, and dotted line refers to changes) : 
(1) Neither physical space nor toponymical words have changed. This situation is dominated by natural or humanistic geographical entities such as mountains, bridges, buildings and so on. As long as the entities still exist, it is more likely that their names will continue to be used. As for referring to the administrative zones of geographic names, it is almost impossible to have an administrative name without any adjustment in entity. Because of past dynasties, political changes are more complicated. Therefore, this study suggests that a political zone only has some parts (such as the boundary of the marginal adjustment or the part system not cutting out to set up administrative zones at the same level, etc.). The toponymal words that remain as themselves can be considered as this category, such as Penglai County, Ju County in Shandong Province, Wuqiang City and Zunhua City in Hebei Province in China

(2) The disappearances of physical space and the drop of toponymal words. Historical geographic names reflect the natural and humanistic features under a certain historical period and geographic environment. With the developments of history, these natural and humanistic features have changed. The original physical space which are closely related to historical geographic names have disappeared and their disappearances make the relevant toponymal words no longer used. Among geographic entity names, the physical space which refer to geographic changes are the most common phenomenon in history, such as buildings, streets and lanes in river and lake water bodies and urban settlements. Therefore, it is the most common situation that the names of river systems, landmark buildings, streets and lanes are no longer used due to the disappearance of physical space. Among geographic names, administrative areas are common, such as the Province of the Yuan Dynasty. As a result of the re-establishment of the Ming and Qing Dynasties, the physical space of several provinces of the Yuan Dynasty did not exist and most of their names were no longer used, such as Zhemin Province, Shangan Province, Huguang Province and so on. Another famous example is Loulan City. The ancient city of Loulan was abandoned because it was cut off from water. Archaeological excavations in the early 20th century brought the city back to life but the glories of Loulan can only be seen in the literature.

(3) The physical space is partially changed but the toponymal words are still in use. Compared with the first two cases, the relationship between "Name" and "Entity" is more complicated. In the previous two kinds of evolution, the "Name" and "Entity" has always been strict and there are no big changes. This kind of historical geographic name, in fact, has some obvious changes which have taken place in different periods but the change is still limited in a certain range, making the "Name" and "Entity" correspondent without ambiguity. So the names can still refer to the same objects. This situation is most typical in the names of political districts. Specifically, in terms of political places, though it is likely to have shrinking space range happens (such as cutting out parts of the edge area to set up new administrative zones at the same level, etc.), but its capitals (that is, the centers are located) do not change still in the administrative zones. Centers generally do not change or even migrate, which are still in the scope of administrative zones, such as Yuhang, Xiaoshan in Hangzhou, Zhejiang Province, etc. As far as geographic names in geographic entity type are concerned, its core areas remain corresponding with the names. Despite its expanding or shrinking range, it will not affect the referring relationship of the names.

However, if the variations exceed a certain limit, great changes would happened to the entities(such as a big adjustment in political space, the original name of the administrative center is no longer referring to the scope of this administrative zones, etc.), referring to another object, which does not belong to this kind of situation; In other words, it should be renamed. If the original names are still used, there may be some confusion; Of course, in the actual management of geographic names, we should try to avoid this situation.

(4) The physical space is basically unchanged but parts of toponymal word changed (that is, the word has the same meaning before and after the change and the latter comes directly from the 
former or derives from the former). This type of situation is mostly seen in cases of geographic names from oral form to written form, transcription gradually returning to a word or original words refining to a better ones. Also, there are errors due to confusion and vulgarization. For example, Fusujing Street in West District, Beijing, whose original name was "Kushuijing Street", for the reason that there was a well with very bitter water? Beijing's Zhongguancun, formerly known as Zhongguancun .It is said that there was a cemetery where many of the Ming Dynasty's eunuchs were buried and called Zhongguancun 中官村. Geographic names sometimes become better, sometimes vulgarized. For vulgarization, there is a famous example. A well-known poet in Tang Dynasty, Bai Juyi, mentioned a place called Hamaling in The Song of a Lute where was inhabited with prostitute in the Tang Dynasty. The geographic name was changed from Xiamaling where was the tomb of Confucian Dong Zhongshu in Han Dynasty, at that time scholars should dismount to show respect for him. So this place name was Xiamaling, meaning a place to dismount. Because of the long time, the place name misread into "Hamaling".

(5) The physical space is basically unchanged but the historical geographic names are completely abandoned. This is the classic case of renaming. Due to the needs of social, economic, political and other factors or due to the changes of the entities themselves (the spatial scope is shrinking or the content and attributes of the natural and cultural landscape are changed) or even disappear, the original names are no longer used, replacing with new ones. Especially at present, with the development of modernization and urbanization, which is the reconstruction of old cities and the demolition of historic buildings, new buildings and expansion roads require various new names. To some extent, developers and governments may abandon the old names for various purposes. So this situation is more common in all six types. The most typical example is the Shaoxing Prefecture in the early Southern Song Dynasty, which replaced Kuaiji Prefecture. Huangshan in Anhui Province replaced Huizhou in nowadays. This situation often leads to the rupture even disappearances of the original history and culture of these places. If the name of Huizhou is abandoned, Huizhou culture has been broken to a certain extent and the new Huangshan culture still needs time to develop (of course, there are other aspects such as the influence of administrative division adjustment).

(6) The physical space is completely changed but the toponym words do not change. This situation is more special. In fact, the phenomenon of so-called "place name migration", often results from some special reasons. For example, during the Eastern Jin Dynasty and the Southern Dynasty in history, setting of emigrant state and county was a large-scale phenomenon of geographic name migration which is caused by the large number of people moving from the north to the south. For another example, Dinghai District in Zhoushan City of Zhejiang Province was once called Changguo County (in 1073) in the Northern Song Dynasty then the system was abolished. When the county was re-established in 1687 (1687) by Emperor Kangxi, it was then renamed as Dinghai County, changing the used Dinghai County to Zhenhai County. In this way, the name of Dinghai County has not changed but the physical space has completely changed. As for the term "Huizhou" can be considered as this category, from the scope of Huizhou government in Ming and Qing Dynasties to the scope of Huizhou District in Huangshan City in today. In terms of the change, there will be some confusions in the short term. So it takes a long time to re-establish the new correspondence, cognition and connotation of the words and entities.

Generally speaking, in the first three cases of the evolution of historical geographic names, this study consider the corresponding relationship between "name" and "entity" is clear and the connotation of geographic names is clear with the reference object basically the same. That is, the phenomenon of renaming has not occurred. In the last three cases, the correspondences between "name" and "entity" has changed, that is, the connotation of geographic names has changed, so it is often accompanied by the process of renaming. 


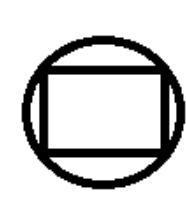

(1)

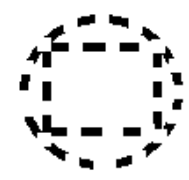

(2)

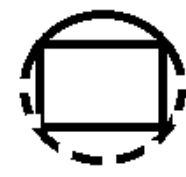

(3)

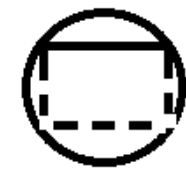

(4)

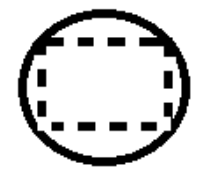

(5)

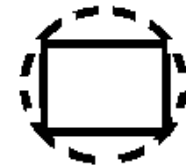

(6)

Figure 1. Six situations of the evolution of historical geographic names

\section{Protection of Historical Geographic Names in China}

Historical geographic names have practical significance, academic significance and even political significance, such as safeguarding national territorial sovereignty. In view of these considerations, the international community has begun to pay attention to the protection of historical geographic names. The United Nations has issued a variety of resolutions related to the protection of geographic names. There are many measures for the protection of historical geographic names in some countries, such as the database of historical geographic names that is being established in the Netherlands and northern European countries regarding historical geographic names as an important part of their cultural heritage. In China, the protection measures, such as the "Chinese toponym cultural heritage protection project", which is being implemented gradually, are also being pushed forward.

According to the development and evolution rules of historical geographic names and relevant experience at home and abroad, the protection of historical geographic names can be promoted from the following three aspects:

(1) On the basis of an accurate understanding of the relationship between "Name" and "Entity" of the historical geographic names, the government needs a correct treatment of the reservation, usage, discard and replacement of historical geographic names. At the same time, try to keep the certainty and integrity of the geographical entity in historical geographic name as well as the consistency of the correspondence between these two and keep the geographic name.

From the analysis of relationship between "Name" and "Entity" of the geographic names, the consistency of "Name" and "Entity" of the geographic names is the key to maintaining the long life of the historical geographic names. If the "Entity" (geographic entity) changes, the changes of the geographic names will happen sooner or later (otherwise, the relationship between "Entity" and "Name" will be mislead, resulting in confusion of concepts). Therefore, historical geographic names are not necessarily fixed or unchanged. If the entity changed, of course, a new name should be used to represent it. Because of this dialectical relationship, historical geographic names are not unchangeable. Once the entity changed, the geographic names themselves should be adjusted accordingly. That is to say, in the protection of historical geographic names, the issue of retention and renaming cannot be viewed mechanically, rigidly and absolutely. They should have objective understanding.

Of course, if the changes of geographical entity are within certain limits, it will not affect its certainty of the name and corresponding relationship between entity and name (such as the third situation in evolution of historical geographic names), in this case, the government should try to retain and use historical geographic names rather than abandon or alter them.

Thus, in turn, in order to better protect the historical geographic names, people should try to keep the certainty and integrity of the geographical entity (at least, the core scope or connotation of the 
place names should not change dramatically) so that the continuation of the historical geographic names has a solid basis. Therefore, the protection of the related geographical entity has become the premise of protecting historical geographic names to some extent. In this way, national departments are also required to effectively protect and inherit the tangible and intangible heritages of local natural, social, political and cultural heritages so as to guarantee the certainty of the geographic entity and maintain the relatively consistent correspondence between name and entity. Without doubt, this requires the joint efforts of the whole society especially the relevant government departments.

For the management department of geographic names, we should try to make the name and entity correspond to each other. That is, in line with the first three cases (at least the third) and tries to avoid the occurrence of the latter three cases; In other words, the corresponding relationship between name and entity should be kept as far as possible or the whole corresponding relationship and the geographic name should not be changed as far as possible. Especially in the process of political district adjustment, it is necessary to avoid the occurrence of some negative effects caused by easy renaming [12].

(2) From the point of a long historical period, with the social, economic, political and other various aspects of development, geographic entity or regional change is inevitable and then the change of name words is also inevitable. Not all the historical geographic names are able to preserve. There is always a group of abandoned historical geographic names. Hence, in order to avoid the historical messages dismiss which results from geographic name abandoned or entity changed, it is of high necessity to establish various systems in different regions and types of the historical geographic names to keep words culture and physical culture.

Consequently, from the perspective of the protection of historical geographic names, the urgent task at present is to establish a legal and institutional system of "historical geographic names registration", to systematically collect, sort out and compile historical names in a region. On this basis, historical geographic names can be classified and evaluated and protection grade can be divided to establish systems such as protection list, heritage list that can be incorporated into a certain protection system. In practical work, those with high cultural value (including word value, physical value, etc.) should be used continuously or in some way. If the value is low, on the premise of fully recording all kinds of information, it can be adjusted (renamed) as the entity changes, but the new name should also connect with the original content as much as possible.

(3) On the basis of all kinds of historical geographic names information, try to take a variety of ways or actions to activate historical places, namely, by continuous usage and directly compiling to maintain its vitality. And when a number of important historical geographic names have disappeared, the government could establish nameplate or other ways to show the site. A cultural event can be held to promote the historical geographic names and promote it as a daily action.

Besides, it is a great method to combine tourism with historical geographic names and enhance the popularity of historical geographic names by carrying out tourism activities or even directly forming some tourist attractions through geographic names. For example, Hangzhou can enrich the tourism activities by implementing and reproducing the connotation of some historical geographic names in the tourism planning, such as display and sale of centralized screen in "Screen Street" and the reappearance of the scene of vigorous iron playing in the past in "Datieguan", so as to enrich the geographic names and make them full of cultural significance. It can also connect the old geographic names that are abolished in history with tourist attractions. Most of these historical geographic are extracted by poets and literary men, so they are fresh and free from vulgarity and meaningfulness [9]. In the tourism planning, these geographic names are consciously used to integrate into the design of scenic spots and combined with the landscape planning to realize the cultural artistic conception which can maximize the continuation of historical context as well as the 
continuation or revival of the life of historical geographic names.

\section{Conclusion}

As an important national and local heritage, historical geographic names are largely abolished for various reasons, while the importance of historical geographic names makes it imperative to protect them. The arrangement and protection of historical geographic names can provide information for today's researches on the past political developments, social developments and human developments as well as experience for today's administrative division reform. In addition, knowing the historical geographic names can safeguard national territorial sovereignty. For the protection of historical geographic names, we can either take direct measures to establish the protection systems or popularize them in connection with tourism in practice or indirectly protect historical geographic names to continue to exist.

\section{References}

[1] Chen Qiao-yi, The Study on the Dialect Geographic Names in Zhejiang Province [J], Hangzhou: Zhejiang Academic Journal, 1983, 2, p56-61. (In Chinese)

[2] Yin Jun-ke, The Study on the Status of Historical Geographic Names in Toponymy-Take Beijing's Historical Geographic Names as Example [J], Beijing: Social Science of Beijing, 1989, 1, p85-92. (In Chinese)

[3] Yan Zhi-jie, The Analysis on the Function of Historical Geographic Names [J], Shenyang: China Place Name, 1998, 1, p9. (In Chinese)

[4] Chen Qiao-yi, The Study on Geographic Names in Ancient China [J], Shenyang: China Place Name, 2000, 5, p4-6.

(In Chinese)

[5] Chen Qiao-yi, The Study on Geographic Names in Ancient China II [J], Shenyang: China Place Name, 2000, 6, p4-6.(in Chinese)

[6] He Ling, An Initial Study on the Names of the Historical Places in Western Regions [J], Lanzhou: NorthWest Minorities Research, 2005, 1, p48-54. (In Chinese)

[7] Yue Sheng-yang, Ye Chang-hong, The Protection of Historical Place Names in the Management of Beijing[J], Beijing: Social Science of Beijing,2009,6,p77-82.(in Chinese)

[8] Wu Peng-fei, The Study on the Survival Dilemma and Countermeasures of Place Names in Ancient Capitals under the Background of Urban Modernization[J],Yangzhou: China Ancient City,2011,9,p38-42.(in Chinese)

[9] Lin Yu-jun, Zhang Qing-hua, The Study on the Use and Protection of Historical Geographic Names [J], Shenyang: China Place Name, 2013, 6, p20-21. (In Chinese)

[10] Fan Jin-zhao, Zou Lv-hui, The Development and Evolution of the Generic Names and the Characteristics of the Generic Names in Contemporary Urban Areas, Shenyang: China Place Name [J], 2015, 2, p29-31. (In Chinese)

[11] Jin Er-gang, Zhang Wen-fan, Administrative Division and Management of Geographic Names [M], Beijing: China Society Press 1996, p297. (In Chinese)

[12] Fan Jin-zhao, Zhang Jin-ling, Liu Ying-jun ,The Influence of the Reform of the System of Administrative Division on the Conversation of Heritage and its Authenticity in Contemporary China[J], Beijing: Economic Geography, 2009,9,p1558-1564. (In Chinese) 